\title{
ORIGINAL
}

\section{LA EVOLUCIÓN DE LAS REPRESENTACIONES SOCIALES SOBRE LA SALUD DE LAS MUJERES MADRILEÑAS, 1993-2000}

\author{
Fernando Conde y Concha Gabriel
}

\section{RESUMEN}

Fundamentos: En 1993 se realizó una primera investigación entre las mujeres madrileñas. En el año 2000 se llevó a cabo una segunda con el objetivo de analizar la posible evolución de las representaciones sociales sobre la salud en las mujeres y, en consecuencia, reorientar, si fuese necesario, los programas de salud definidos en 1993 en función de los nuevos resultados de la investigación. De esta forma, se pretendía analizar la posible evolución de los discursos, de las representaciones sociales sobre la salud que pudiera expresar entre 1993 y el 2000 cada uno de los cinco tipos encontrados en el 1993.

Métodos: La investigación cualitativa en el 2000 se basó en la realización de 10 grupos de discusión, definidos en función de las «tipologías» de mujeres que se habían construido en la investigación de 1993. La caracterización de los tipos se realizó en función de las distintas variables sociodemográficas y de rol que se habían expresado más importantes en 1993. El análisis de los discursos se tradujo en una forma gráfica, topológica para visualizar la citada evolución de las representaciones sociales.

Resultados: La investigación puso de manifiesto importantes cambios entre 1993 y el 2000. En el 2000 la citada perspectiva discursiva había evolucionado del rol de «ama de casa» al rol de «mujer trabajadora»; en el 2000 las representaciones sociales dominantes hacían hincapié en una dimensión más cercana al equilibrio personal en lo que el papel de la propia mujer, más allá de su familia, tiene mucha importancia; el ámbito de la preocupaciones también evolucionaba, pasando al estrés por la doble jornada laboral

Conclusiones: El estudio permitió obtener conclusiones de tipo metodológico, vinculadas a la capacidad de la metodología cualitativa para hacer estudios comparativos y temporales, y conclusiones de carácter más pragmático, que permitieron reorientar determinados programas de salud orientados a las mujeres en una línea más cercana a sus preocupaciones.

Palabras clave: Investigación cualitativa. Salud de la mujer. Grupos de discusión.

\section{Correspondencia:}

Fernando Conde

Cimop

Sagasta, 28

28004 Madrid

Correo electrónico: fernandoc@retemail.es

\section{ABSTRACT}

The Evolution of the Social Representations Regarding Health of Women from Madrid, Spain, 1993-2000

Background: In 1993, initial research was conducted among the women in Madrid. In 2000, a second research was conducted for the purpose of analyzing the possible evolution of the social representations related to health among women and, if necessary, to therefore reorient the health programs set forth in 1993 in terms of the new results of the research. The aim was therefore to analyze the possible advancement of the opinions stated, of the health-related social representations which each one of the five types encountered in 1993 might express throughout the 1993-2000 period.

Methods: The qualitative research conducted in 2000 was based on the holding of 10 discussion groups defined according to the women's «typologies» which had been set out in the 1993 research. The types in question were characterized in terms of different sociodemographic variables and of the role which they had stated as most important in 1993. The analysis of the stated opinions was put into graphic and topological form in order to display the aforementioned trend in the social representations

Result: This research revealed some major changes having taken place throughout the 1993-2000 period. the predominan stated opinion-related perspective had evolved out of the «housewife» role to the «working woman» role in 2000. the predominant social representations stressed a dimension more nearing a persona balance in 2000, in which the role of women themselves, outside the bounds of their families, is of major importance. The scope of their concerns also evolved to a concern related to the stress involved in being a working woman plus a housewife.

Conclusions:. This study made it possible to reach conclusions of a methodological type related to the ability of the qualitative methodology to make comparative, time-based studies and to conclusions of a more pragmatic type which afforded the possibility of changing the orientation of certain health programs aimed at women along a line more closely in keeping with their true concerns.

Keywords: Qualitative research. Women's health. Discussion groups. 


\section{INTRODUCCIÓN}

En el año 1991, la Dirección General de Salud Publica de la Comunidad Autónoma de Madrid inició un programa de investigaciones con el objetivo de construir un «Sistema de Indicadores Socioculturales sobre la Salud», tal como se desarrolla en estas mismas páginas en el artículo de $\mathrm{M}$ Gil Nebot ${ }^{1}$. La gran mayoría de los indicadores de salud disponibles adoptan, como es sabido, la forma de tasas $^{2-5}$ definidas y construidas por los profesionales $\mathrm{y} / \mathrm{o}$ autoridades sanitarias, sin tener en cuenta las posibles concepciones de la salud que pudieran tener los distintos grupos sociales de una sociedad determinada. En algún caso, se ha tratado de definir algún nuevo tipo de indicadores denominados «blandos», que sofisticando técnicamente su proceso de construcción no modifican, sin embargo, la cuestión de fondo de la definición de los mismos ${ }^{6}$. El programa del «Sistema de Indicadores Socioculturales sobre la Salud» partía, sin embargo, del principio de que dicho sistema debía construirse a partir de lo que los grupos sociales, caracterizados como «biosocioestratos» en las bases teóricas del programa, pueden entender como «salud». Se planteó, por ello, la necesidad y la posibilidad de construir los «indicadores socioculturales» de una forma más abierta que la tradicional, partiendo de una aproximación cualitativa y tratando de complementar dicha aproximación con los resultados procedentes de la metodología cuantitativa.

El objetivo de todo ello era el de disponer de una herramienta «técnica» que facilitara la elaboración de políticas y estrategias de intervención en el ámbito de la salud publica y que, por tanto, posibilitara mejorar el diseño de los distintos programas de promoción de la salud que se venían realizando en la Comunidad de Madrid. Asimismo, se pretendía poder analizar la evolución en el tiempo de las concepciones sobre la salud de la sociedad madrileña, de modo que se pudiera ir renovando y actualizando el citado diseño de los programas de salud, en fun- ción de la propia evolución de la sociedad a este respecto.

Para responder a esta demanda acudimos a la teoría de las representaciones sociales, como el marco teórico que nos parecía más adecuado para el planteamiento y desarrollo de la investigación propuesta ${ }^{7,8}$, y a una línea de trabajo metodológica que descansaba en la articulación de las técnicas cualitativas y cuantitativas que habíamos tenido ocasión de proponer y desarrollar en años anteriores $^{9,10}$. Línea de trabajo que está explicitada en el primer volumen, informe mujer y salud $^{11}$, del conjunto de monografías que componen el desarrollo de este programa de investigaciones y que están, en su práctica totalidad, publicadas en la colección de Documentos Técnicos de Salud Pública de la Consejería de Sanidad y Consumo de la Comunidad Autónoma de Madrid en los números 32, informe mujer y salud ${ }^{11}$ en el 45 , los jóvenes y la salud ${ }^{12}$; en el 47 , los varones adultos y la salud ${ }^{13}$; en el $48, \operatorname{los}$ niños de 6 a 12 años y la salud ${ }^{14}$; y en el número 50 , los mayores y la salud ${ }^{15,16}$.

Posteriormente, en el año 2000, se planteó la necesidad de actualizar el estudio de las mujeres y la salud en relación con la investigación pionera de 1993, de forma que se pudieran deducir las líneas de trabajo que se considerasen más interesantes a implementar como consecuencia de dicha evolución. De forma secundaria, se trataba de evaluar hasta qué punto los discursos de las mujeres producidos en la investigación cualitativa coincidían con el tipo de problemas de salud recogidos en la investigación clínica y en la investigación epidemiológica que, con otros instrumentos de medida, venía estudiando y analizando la Consejería de Sanidad de la Comunidad Autónoma de Madrid (de esta segunda cuestión, no hacemos mención en este artículo).

La investigación de 1993 estaba compuesta de una fase cualitativa, centrada en la realización de 7 grupos de discusión, y de una posterior fase cuantitativa, basada en 
una encuesta representativa a 1.299 mujeres madrileñas de 18 y más años ${ }^{1}$ En el estudio del 2000, por distinto tipo de razones, de acordó realizar sólo la investigación cualitativa. Investigación cuyo planteamiento, desarrollo y resultados constituye la base de este artículo.

\section{SUJETOS Y MÉTODOS}

Diseño de los grupos. En el año 1993, la realización de la investigación permitió configurar un conjunto de discursos sobre la salud que, posteriormente en el estudio cuantitativo, fueron utilizados como base para construir, mediante la utilización del denominado análisis clusters ${ }^{16}$, una tipología de mujeres madrileñas en función de las concepciones sobre la salud que hubieran evidenciado. Dicho análisis configuró cinco «tipos» de mujeres que denominamos como «mujeres con nociones biomédicas» sobre la salud, que agrupaba un $21,5 \%$ de la muestra, es decir, de mujeres que conciben la salud de una forma muy similar al discurso dominante en las instituciones sanitarias y que reduce prácticamente la salud a la ausencia de la «enfermedad» orgánica; «mujeres preocupadas por su salud» en el $20 \%$ de la muestra, es decir, mujeres que teniendo cierto tipo de problemas de salud, priorizan en sus discursos la preocupación por las enfermedades más allá de cualquier elaboración discursiva más general; «mujeres con nociones holísticas» en un $24,5 \%$ de la muestra, es decir, de mujeres que conciben la salud de una forma muy global e integral, casi como una forma de estar y de sentirse bien en la vida; «mujeres con nociones basadas en el equilibrio personal» con una representación del $17 \%$, corresponde a las mujeres que entienden la salud como un proceso dinámico y flexible en el que los autocuidados ocupan un papel muy importante; por último, «mujeres con nociones basadas en el código del aguante» con otro $17 \%$ de la muestra, es decir, de mujeres que entienden la salud como posibilidad de hacer las tareas y actividades cotidianas, más allá de la presencia o no de enfermedades y que, por la presión del entorno, no declaran el sentirse mal, con tal de seguir realizando dichas tareas.

En el año 2000, para poder permitir la comparación con los resultados de 1993, el diseño de los grupos de la investigación cualitativa planteada se fundamentó en la citada tipología de mujeres construida en 1993. El diseño de los grupos de una investigación cualitativa, como es sabido, no pretende reproducir una determinada situación demográfica, sino configurar una conjunto estructural de grupos que produzcan aquellos discursos que se consideren más relevantes con respecto al objeto de la investigación ${ }^{17,18}$. Por ello tomamos como categorías del diseño de los grupos las variables y las situaciones de rol, de edad, de situación social y de actividad que en 1993 se habían manifestado como más decisivas, desde el punto de vista más estructural, para configurar los citados tipos (en el estudio cuantitativo) y producir sus respectivos discursos (en el estudio cualitativo). Con estas premisas, el diseño concreto de los grupos realizados fue el siguiente:

Reunión de Grupo n. ${ }^{\circ} 1$ (RG1). Mujeres de 25 a 32 años. Solteras y sin hijos. Activas en empresas de servicios con contratos temporales. Clase media y media baja. Fuenlabrada. Se realizó el 21 de noviembre de 2000. La expectativa del diseño era que se produjeran unos discursos cercanos a los denominados «biomédico»y «equilibrio personal».

Reunión de Grupo n. 2 (RG2). Mujeres de 27 a 35 años. Casadas o con pareja. La mitad con hijos y la otra mitad sin hijos. Activas. Ejecutivas de empresa, profesionales autónomas. Clases medias y medias altas Madrid. Se realizó el 7 de noviembre de 2000. La expectativa del diseño era que se produjeran unos discursos cercanos a los denominados «biomédico» $\mathrm{y}$ «equilibrio personal».

Reunión de Grupo n. 3 (RG3). Mujeres de 25 a 30 años. Solteras y sin hijos. 
Estudiantes o en los primeros empleos. Clases medias altas. Madrid. Se realizó el 22 de noviembre de 2000. La expectativa del diseño era que se produjeran unos discursos cercanos a los denominados «biomédico»y «equilibrio personal».

Reunión de Grupo n. 4 (RG4). Mujeres de 30-40 años. Casadas y con hijos (1 ó 2). Ocupadas. Clases medias. Aranjuez. Se realizó el 28 de noviembre de 2000. La expectativa del diseño era que se produjera unos discursos próximos al «holístico» y al del «equilibrio personal».

Reunión de Grupo n. 5 (RG5). Mujeres de 35 a 44 años. Casadas con hijos (1 ó 2). Activas en el sector industrial (textil, química...). Clases medias bajas. Alcalá de Henares. Se realizó el 14 de noviembre de 2000. La expectativa del diseño era que se produjeran unos discursos próximos al «holístico»y al de «equilibrio personal».

Reunión de Grupo n. ${ }^{0} 6$ (RG6). Mujeres de 38-48 años. Casadas y con hijos, la mitad 1 ó 2, y la otra mitad 3 ó más. Activas (funcionarias, profesionales liberales...). Clases medias altas. Madrid. Se realizó el 13 de diciembre de 2000. La expectativa del diseño era que se produjeran unos discursos próximos al «holístico»y al de «equilibrio personal».

Reunión de Grupo n. 7 (RG7). Mujeres de 37 a 45 años. Casadas y con hijos, la mitad 1 ó 2, y la otra mitad 3 ó más. Amas de casa. Clases medias. Móstoles. Se realizó el 29 de noviembre de 2000. La expectativa del diseño era que se produjeran unos discursos próximos al «holístico» y al del «código del aguante».

Reunión de Grupo n. ${ }^{\mathbf{0}} 8$ (RG8). Mujeres de 55 a 65 años. Casadas y con hijos, la mitad 1 ó 2, y la otra mitad 3 ó más. Amas de casa. Clases medias y medias altas. Majadahonda. Se realizó el 8 de noviembre de 2000. La expectativa del diseño era que se produjeran unos discursos próximos al «holístico» y al de «preocupadas por la salud».
Reunión de Grupo n. 9 (RG9). Mujeres de 45 a 55 años. Casadas y con hijos, la mitad 1 ó 2, y la otra mitad 3 ó más. Amas de casa y ocupadas. Presencia de alguna enfermedad. Clases medias. Madrid. Se realizó el 30 de noviembre de 2000. La expectativa del diseño era que se generara un discurso próximo a los discursos «preocupados por la salud».

Reunión de Grupo n. ${ }^{\mathbf{0}} \mathbf{1 0}$ (RG10). $\mathrm{Mu}$ jeres de 55 a 65 años. Casadas y con hijos, la mitad 1 ó 2, y la otra mitad 3 ó más. Amas de casa. Clases medias y medias bajas. Barrios periféricos de Madrid. Se realizó el 21 de noviembre de 2000. La expectativa del diseño era que se generaran unos discursos próximos al del «código del aguante» y al de «preocupadas por la salud».

Conjunto de grupos que, en función de su posición en relación con unos ejes de estatus y de «modernización» en lo que a sus estilos de vida se refiere, constituyen el Mapa de Grupos reproducido en la figura 1.

El impulso inicial de los grupos realizados: En la misma línea de garantizar, en la medida de lo posible, unas condiciones de producción de los discursos similares a las de 1993, el impulso que se planteó a los grupos del 2000 fue el mismo que en 1993, esto es, que se estaba realizando una investigación para conocer que es lo que se entiende por calidad de vida, por bienestar, por sentirse bien, por estar bien. Es decir, no planteamos ninguna expresión directamente equivalente al término "salud», de forma que pudiéramos observar cómo y de qué forma surgía dicha noción en el devenir espontáneo de la dinámica de los grupos. Para garantizar este objetivo, las dinámicas de grupo se desarrollaron de forma abierta y no directiva. Solamente al final de las mismas, cuando los grupos habían desarrollado sus discursos y sólo en el caso de que no hubieran surgido espontáneamente, el moderador, de forma más directiva y sugerida, planteaba a los grupos que desarrollaran sus opiniones sobre ciertos programas de salud que in- 


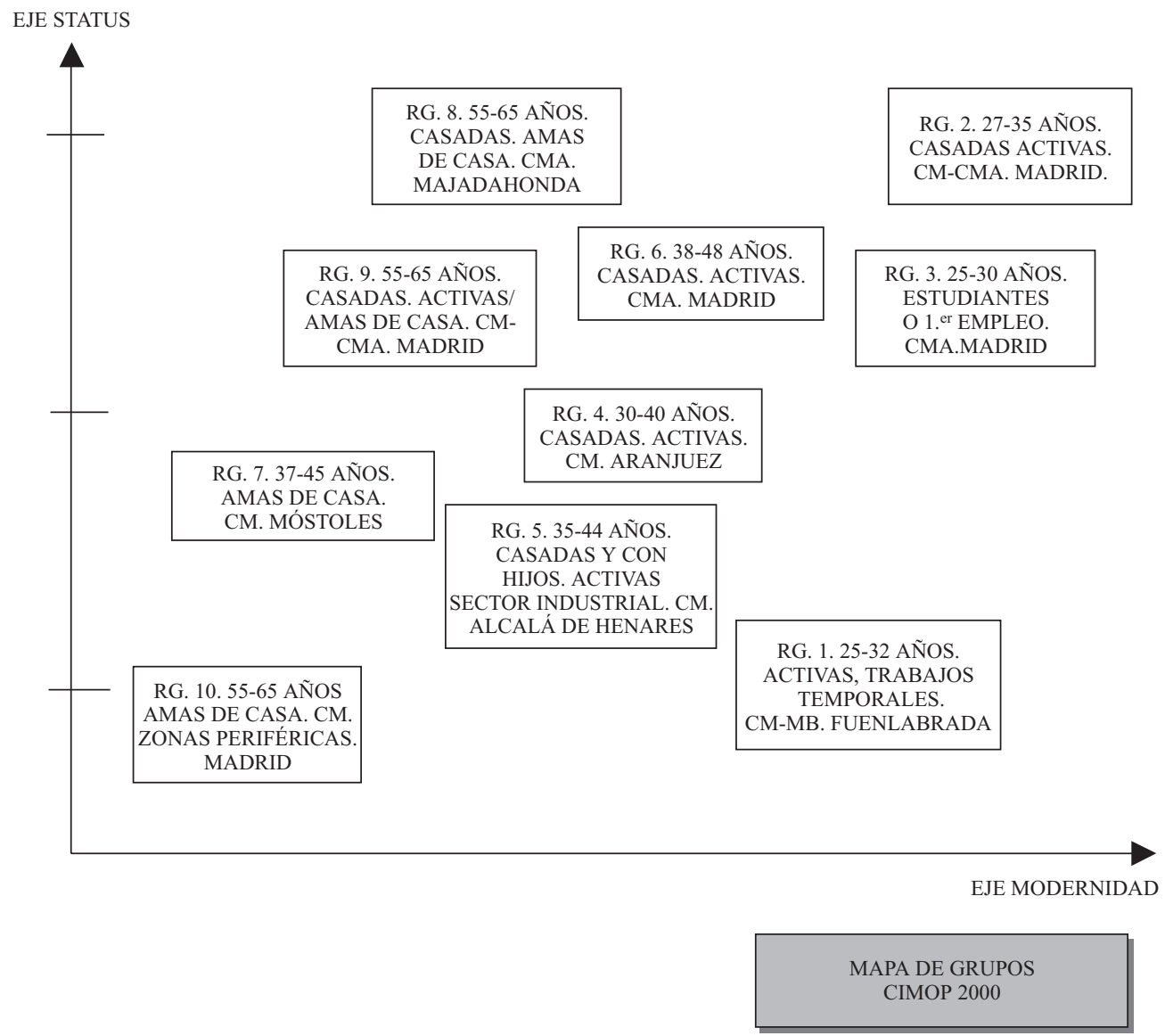

teresaba investigar y sobre algunas de las «enfermedades» que, según las estadísticas sanitarias, más afectan a las mujeres.

La moderación de los grupos fue llevada a cabo por una de las autoras del trabajo, para facilitar la producción de un discurso más libre que el que se hubiera podido producir delante de un hombre. El trabajo de análisis e interpretación fue realizado por los dos autores que firman el artículo.

\section{RESULTADOS}

Aunque en la investigación surgieron y se desarrollaron diversos temas, en este artículo sólo vamos a recoger aquellos aspectos de la investigación más directamente relacionadas con los objetivos centrales de la misma y, en especial, los que se refieren a la evolución de los discursos. Desde esta perspectiva, los discursos producidos por las mujeres madrileñas en el 2000 presentan los 
siguientes perfiles de evolución temporal en relación a los de 1993.

1. La evolución de los «estilos discursivos» En 1993, los discursos de las mujeres vinculaban expresivamente unas y otras temáticas de la salud en el marco de un desarrollo conversacional, de un «habla», de unos argumentos que iban entretejiendo y entreverando sintagmáticamente unos y otros tópicos en un «estilo discursivo» que algunos autores han denominado como «femenino». Sin embargo, los discursos del 2000 diferenciaban más unos y otros temas, separaban más el abordaje de unas y otras temáticas, generando un discurso estilísticamente más próximo, en algunos casos, al producido por los varones en el estudio llevado a cabo en 1995, que al elaborado por las mujeres en 1993. Mientras en este último año, los distintos temas tratados formaban una especie de cadena en la que los diferentes tópicos sobre la salud estaban unidos como eslabones de la misma, en expresión de uno de los grupos realizados, en el 2000 , la citada cadena se había roto y los eslabones de 1993 se habían convertido en tópicos más aislados, más separados unos de otros.

2. La evolución de la «perspectiva discursiva», es decir, de la posición desde la que se elaboran los discursos en los grupos. En 1993, el espacio matriz en el que se tendían a producir los discursos femeninos sobre la salud era el espacio asociado al rol maternal, en el año 2000, sin embargo, dicho espacio matriz se había desplazado a lugares marcados por el desempeño de los roles laborales de las mujeres que trabajan fuera del hogar.

3. La evolución de los «discursos centrales» sobre la salud, es decir, de los discursos dominantes producidos por el conjunto de grupos realizados. En estrecha relación con lo señalado en el punto 2, en 1993 se producían unos discursos que integraban la salud y la enfermedad entendidas en el sentido biomédico más reductor, en una concepción holística más amplia de la que formaban parte la calidad de vida, las relaciones familiares, unas relaciones sexuales satisfactorias, etc., como puede observarse en la figura 2, resultado de un análisis no métrico realizado en la encuesta de 1993 (en el gráfico citado se entiende que los puntos próximos en el mapa, guardan una estrecha relación entre sí). En el año 2000, sin embargo, se producían unos discursos donde las concepciones sobre la salud se expresaban como nociones más centradas en la búsqueda del «equilibrio personal» muy directamente relacionado, desde nuestro punto de vista, con las teorías del «yo en relación» que han desarrollado ciertas corrientes de pensamiento feminista. Noción del «equilibrio personal» a caballo, si se nos permite esta expresión, entre las concepciones más holísticas y familiaristas de 1993 y las más individualistas y biomédicas más presentes y dominantes en las instituciones sanitarias. Evolución que hemos tratado de representar en la figura 3 y que se traducía en que en el 2000 la preocupación central de las mujeres era la atención por su propia salud y los consiguientes cuidados personales, mientras que en el 1993 la preocupación central era la situación de la salud familiar y, en particular, la de los hijos. Una expresión significativa de esta evolución la podemos observar en las mismas expresiones de los grupos. En 1993, las referencias a la vida sana, a la vida saludable, a la familia, etc., atravesaban los discursos grupales, en el 2000, dichas expresiones más globales tendían a disminuir y a ser sustituidas por otras vinculadas a relatos más personalizados en los que expresiones como tener energía, tener vitalidad $\mathrm{u}$ otras muy próximas, ocupaban un lugar central.

En el seno de esta tendencia que atraviesa todos los grupos, cabía, en todo caso, diferenciar entre dos situaciones generacionales muy distintas. En las mujeres de más de 50 años seguían muy fuertes y presentes las nociones sobre la salud analizadas en 1993. En las mujeres de menor edad se expresaban con mucha fuerza las nociones sobre la sa- 
Figura 2

Indicador sociocultural. Mapa de representaciones sociales sobre la salud. Total muestra. Cimop, 1994

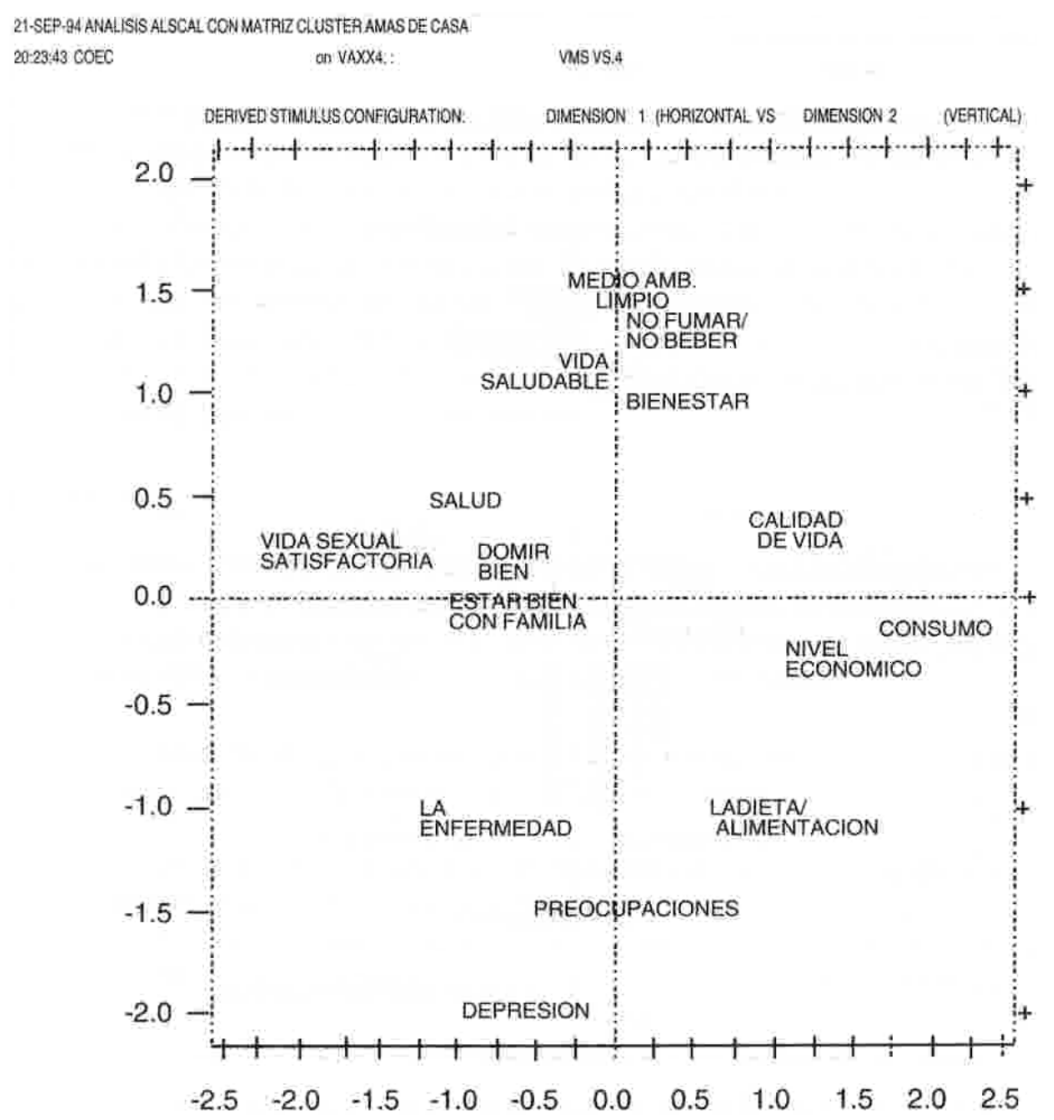

lud más personalizadas y centradas en la búsqueda del «equilibrio personal».

\section{La evolución de las «tipologías» de} mujeres construidas en función de los discursos elaborados. El estudio de 1993, como habíamos señalado anteriormente, posibilitó la construcción de cinco tipos básicos de mujeres madrileñas en función de las principales características discursivas que se habían puesto de manifiesto. En el año 2000, la ausencia de un estudio cuantitativo impidió comparar con precisión el peso so- cial de cada uno de los discursos. Sin embargo, la propia investigación cualitativa permitió construir la hipótesis, a expensas de una posterior contrastación empírica mediante la realización del estudio cuantitativo correspondiente, de que, en el marco de la evolución citada en el punto 3 , se han producido las siguientes tendencias de cambio en el peso social de unos y otros discursos: Incremento del peso social del discurso «biomédico», fuerte incremento del discurso del «equilibrio personal», estabilización del discurso «preocupado por la salud»y 


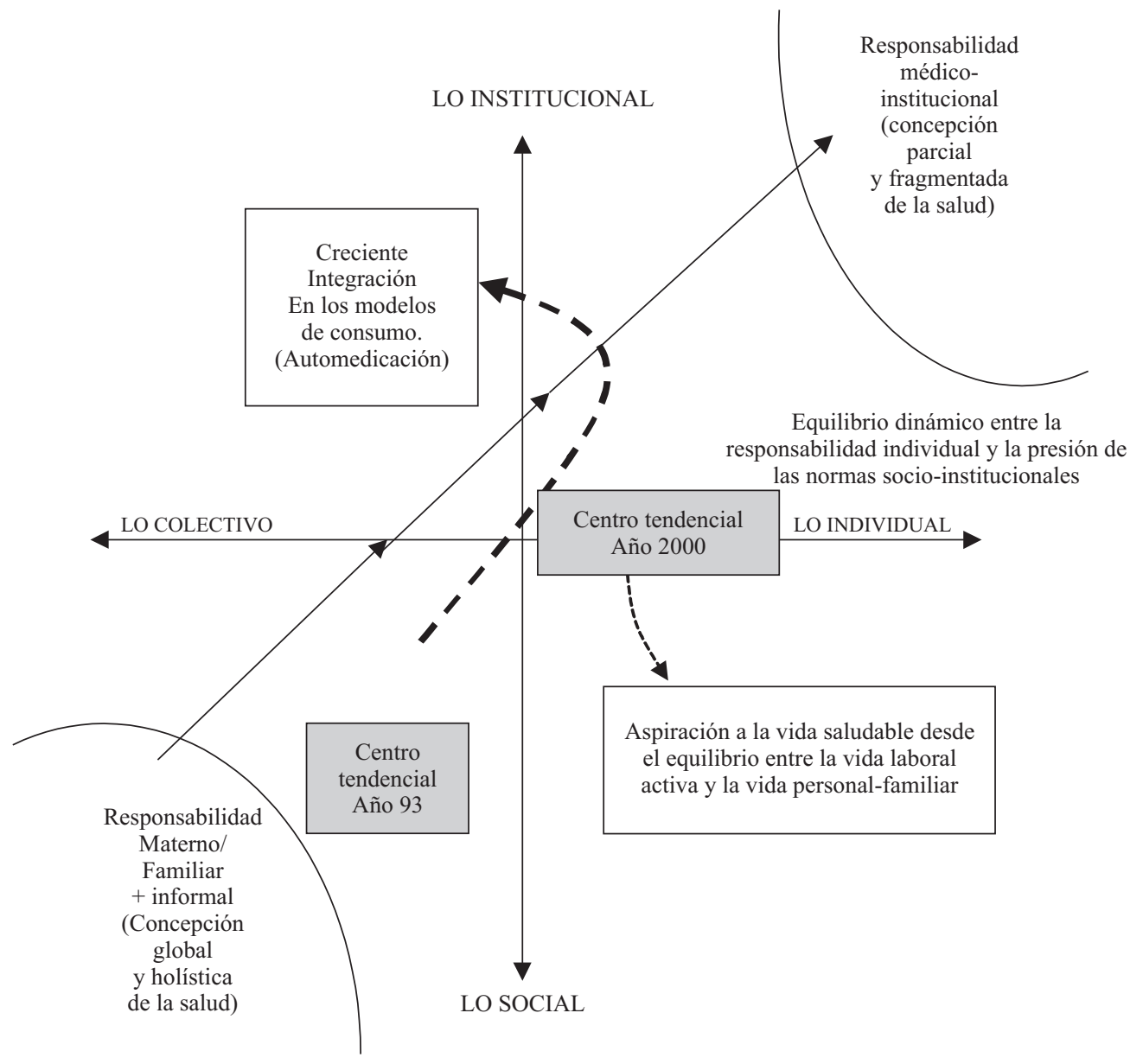

perdida de peso de los discursos «holístico» y del «código del aguante».

5. La evolución de las principales «preocupaciones». En paralelo a la modificación de las nociones y representaciones sociales de las mujeres sobre la salud, se produce también una transformación de las situaciones de malestar espontáneamente más señaladas por los grupos de mujeres. En 1993 la situación patológica más señalada era la depresión y la solución apuntada para salir de la misma era, entre otras cuestiones, salir de la casa, romper con el encierro de las cuatro paredes del hogar familiar. En el año 2000, la situación había cambiado radicalmente. $\mathrm{La}$ depresión seguía apareciendo como un horizonte patológico, como un riesgo posible en aquellos grupos (minoritarios) de una cierta edad pero, sin embargo, el emergente que afloraba con fuerza en la mayoría de los grupos era el estrés, como factor de riesgo aso- 
Figura 4

Evolución de los roles y de las principales situaciones patológicas en las mujeres madrileñas entre 1993 y 2000

\begin{tabular}{|c|c|c|c|}
\hline & SITUACIÓN EN 1993 & \multicolumn{2}{|c|}{ SITUACIÓN EN 2000} \\
\hline $\begin{array}{l}\text { ROLES } \\
\text { DOMINANTES }\end{array}$ & $\begin{array}{l}\text { - Ama de casa «evolucionada». } \\
\text { Rol parcial que no se confunde } \\
\text { con la identidad. }\end{array}$ & \multicolumn{2}{|c|}{$\begin{array}{l}\text { - Mujer activa y trabajando fuera del hogar. } \\
\text { - Continuidad de la responsabilidad y el ejercicio de las tareas } \\
\text { de la casa. } \\
\text { - Doble jornada. }\end{array}$} \\
\hline $\begin{array}{l}\text { SITUACIONES } \\
\text { ASPIRACIONALES }\end{array}$ & $\begin{array}{l}\text { - Roles y tareas laborales. El } \\
\text { «mundo del afuera» como posi- } \\
\text { tivo y saludable. }\end{array}$ & \multicolumn{2}{|c|}{$\begin{array}{l}\text { - Encontrar el equilibrio entre las distintas demandas. } \\
\text { - Articular esfera laboral, familiar y personal. }\end{array}$} \\
\hline $\begin{array}{l}\text { REFERENCIAS } \\
\text { CULTURALES } \\
\text { DOMINANTES }\end{array}$ & $\begin{array}{l}\text { - Modelo patriarcal que asigna a } \\
\text { la mujer al rol de Madre. }\end{array}$ & \multicolumn{2}{|c|}{$\begin{array}{l}\text { - Modelo de desarrollo personal más autónomo en el marco del } \\
\text { modelo social de consumo. }\end{array}$} \\
\hline $\begin{array}{l}\text { PRINCIPAL } \\
\text { PATOLOGÍA } \\
\text { ASOCIADA }\end{array}$ & - Depresión. & \multicolumn{2}{|l|}{ - Estrés. } \\
\hline $\begin{array}{l}\text { CARACTERIZACIÓN } \\
\text { PATOLÓGICA }\end{array}$ & - Enfermedad. & \multicolumn{2}{|c|}{ - Malestar con riesgo de enfermedad. } \\
\hline \multirow[t]{3}{*}{$\begin{array}{l}\text { TERAPIA } \\
\text { SUGERIDA }\end{array}$} & \multirow[t]{3}{*}{$\begin{array}{l}\text { - Salir de la casa y tener activida- } \\
\text { des sociales. } \\
\text { - Medicación bajo control médica } \\
\text { en casos graves, «No hay aspiri- } \\
\text { nas para los dolores del alma». }\end{array}$} & \multicolumn{2}{|c|}{$\begin{array}{l}\text { - Rencontrar energía y equilibrio. } \\
\text { - Controlar las múltiples presiones. } \\
\text { - Automedicación (Complejos Vitamínicos, Ansiolíticos...) }\end{array}$} \\
\hline & & $\begin{array}{l}\quad \text { GRUPOS C. M. BAJA } \\
\text { - Recomponer energía física } \\
\text { • Pausa en la actividad. } \\
\text { - Conseguir ayuda en las tareas } \\
\text { domésticas. } \\
\text { - El hogar y la familia como } \\
\text { espacio de desarrollo. }\end{array}$ & $\begin{array}{l}\text { GRUPOS C. M./M ALTA } \\
\text { • Mantener y promover } \\
\text { la VITALIDAD como } \\
\text { energía y fuerza de ánimo. } \\
\text { • El trabajo y las relaciones } \\
\text { sociales como espacio } \\
\text { de desarrollo. }\end{array}$ \\
\hline & & $\begin{array}{l}\text { Importancia de la energía } \\
\text { ísica. } \\
\text { Abrirse a la importancia } \\
\text { de lo psíquico («arreglarse»). }\end{array}$ & $\begin{array}{l}\text { - Importancia del cuerpo «activo», } \\
\text { «vital» en el que se expresa la } \\
\text { fuerza del espíritu. }\end{array}$ \\
\hline
\end{tabular}

ciado a las nuevas condiciones de vida de las mujeres con doble jornada, evolución que hemos tratado de resumir en la figura 4.

No deja de ser relevante, en relación con esta evolución, que mientras en 1993 el espacio fuera del hogar siempre aparecía connotado por valores positivos, saludables, en el 2000 aparecía marcado de forma ambivalente en la medida que podía ser saludable, en unos casos, y fuente de malestar, agobio, polución, ruidos y estrés en otros. En la dimensión del tiempo ocurría una evolución similar pero en un sentido contrario. En 1993 el tiempo aparecía en los discursos de forma ambivalente por el miedo a los «tiem- 
pos muertos»), a la soledad, mientras que en el 2000 aparecía connotado más positivamente por la posibilidad de tener un tiempo propio, un tiempo para sí misma como condición sine qua non para lograr un cierto equilibrio personal.

Asimismo, en el 2000 en relación con 1993, había disminuido el temor a las enfermedades infecciosas, como el vih-sida. Por el contrario, apareció con mucha fuerza la preocupación por las enfermedades crónicas y/o invalidantes, a modo de contra-figura, de inversión de las nociones del equilibrio personal que tiene en la autonomía personal uno de sus fundamentos. También observamos la disminución de la tolerancia en los umbrales del dolor y una mayor dependencia de los profesionales sanitarios, una vez que la enfermedad ha hecho su aparición.

\section{DISCUSIÓN}

Los resultados de la investigación posibilitan tres líneas de reflexión al respecto. Una más centrada en el propio planteamiento metodológico. Otra centrada en la interpretación de las posibles dimensiones que subyacen en la evolución detectada y, por último, otra más centrada en la utilidad más operativa de los resultados para el diseño de los programas de salud, objetivo último de la investigación realizada.

En relación con la primera cuestión cabe señalar que lo más pertinente en el terreno estrictamente metodológico hubiera sido repetir plenamente la investigación de 1993. Por distintas razones, alguna de ellas señalada por Gil Nebot en estas mismas páginas ${ }^{1}$, esto no pudo realizarse. Sin embargo, la formalización de los resultados de 1993 en figuras que reproducían una cierta estructura de orden en las posiciones de los discursos y en las problemáticas abordadas en los mismos, permitió realizar las comparaciones de los resultados obtenidos en 1993 y en el 2000, tal como hemos tratado de evidenciar en este artículo.
En este sentido, creemos que la investigación supone una demostración de lo injustificado de alguna de las críticas más habituales a la metodología cualitativa cuando se señala su escasa, cuando no nula, posibilidad de formalización y su incapacidad para realizar estudios comparativos ${ }^{19}$ confundiendo, a nuestro juicio, la implicación del investigador en la investigación, el afán comprensivo y no meramente explicativo ${ }^{20}$ de la perspectiva cualitativa con una incapacidad de formalización y de contrastación intersubjetiva. La investigación realizada muestra cómo es posible formalizar topológicamente $^{9,10}$ sus principales resultados $\mathrm{y}$ con ello permitir una contrastación y una comparación con otras metodologías y otras investigaciones. Es decir, en aquellos casos en los que se produce una cierta cristalización discursiva, esto es, una cierta estabilización en la trama conversacional de los grupos y en las cadenas asociativas que éstos producen, es posible formalizar las dimensiones ordinales implícitas que subyacen en dicha cristalización.

Una de las posibles formas de hacerlo, siguiendo los ejemplos de las figuras 2 y 3 , es estructurar un gráfico a partir de unos ciertos ejes, a modo de ejes de coordenadas, que cabe configurar a partir de las dimensiones implícitas en las dinámicas de grupo, y del análisis de los juegos de cadenas asociativas que se producen en las mismas. Por ejemplo, una vez configurados los ejes social-institucional y colectivo individual de la figura 3 , de lo que se trata es de representar el conjunto de expresiones que connotan un mismo significado así como el juego de relaciones de orden existentes entre los mismos. Conjunto de expresiones con la misma connotación que podemos denominar campos semánticos. Por ejemplo, en la figura 2, podríamos entender que los términos medio ambiente, vida saludable, bienestar, no fumar y no beber, configuran un cierto campo semántico; que los términos salud, vida sexual satisfactoria, dormir bien y estar bien con la familia constituye otro y así sucesivamente. Y, a la vez, el juego de relaciones de 
orden existentes entre los cuatro principales campos semánticos representados nos ayudan a interpretar el espacio socio-simbólico en el que se producen los discursos femeninos sobre la salud en el citado año.

No conviene olvidar que la representación topológica de una cierta estructura de variables o de dimensiones, según el caso del que se trate, tal como hemos podido observar en los citados ejemplos de las figuras 2 y 3 , tiene la propiedad de la estabilidad estructural de la forma ${ }^{21,22}$, esto es, tiene la propiedad de ser una representación que produce lo que se denomina una forma invariante, que permanece constante siempre que los parámetros que la conforman no modifiquen las relaciones de orden que puedan existir entre ellos. Desde esta perspectiva, mientras un conjunto de discursos mantengan entre sí las mismas relaciones de orden simbólico, su representación formal permanece invariante y, viceversa, una modificación en la citada representación formal sería un claro indicador de que se han modificado las posibles relaciones de orden existentes entre los discursos representados en dicha forma en la figura. De este modo, la representación topológica de las relaciones de orden entre los discursos producidos en una investigación cualitativa, tal como hemos tratado de apuntar muy sintéticamente en este artículo, permite los estudios comparativos, ya sea entre estudios, ya sea a lo largo del tiempo a partir, precisamente, del análisis de la posible evolución de las citadas representaciones formales o topológicas.

En lo que se refiere a la segunda cuestión, cabe pensar que la evolución discursiva señalada se debe en parte al diseño de la propia investigación, con presencia de un mayor número de grupos de mujeres jóvenes que en 1993. Sin embargo, cabe pensar que el factor esencial asociado a este cambio ha sido la amplia incorporación de las mujeres madrileñas a la vida sociolaboral fuera del hogar, la cual introduce nuevas perspectivas vitales en las mujeres y favorece el desarro- llo de nuevas concepciones y preocupaciones por la salud, distintas a las que se mantienen cuando los roles dominantes son los de «ama de casa».

Por último, en lo que se refiere a la utilidad de la investigación para el diseño de los programas de salud dirigidos a las mujeres cabe señalar las siguientes cuestiones.

En primer lugar, aparece la necesidad de segmentar más claramente los programas de salud en una relación más directa y personalizada con las usuarias potenciales de los mismos. En la práctica, a mediados de los años 90, más allá de los programas de salud reproductiva, una gran parte de los programas de salud existentes en Madrid y, en general, en toda España, implícita o explícitamente parecían destinados a mujeres con una cierta edad y muy cercanas al desempeño de los roles más tradicionales de «ama de casa». Por ejemplo, los programas sobre la menopausia y sobre osteoporosis, muy presentes en las Carteras de Servicios del Sistema Sanitario, estarían muy cerca de esta orientación. Ahora bien, junto con este tipo de programas, ésta investigación demuestra que conviene poner en marcha otras líneas de intervención que se acerquen al resto de las mujeres madrileñas y que lo hagan desde las nuevas perspectivas e inquietudes que las mujeres expresan. No hay que olvidar que un amplio número de mujeres ya no se reconoce en los roles más clásicos de ama de casa, expresando otro tipo de exigencias, más cercanas a la preocupación por su propia situación personal que a la orientación de «cuidadora» más clásica de muchos de los programas de salud destinados a la mujer. En este sentido, cabría señalar la necesidad de cambiar la orientación central de los programas actuales destinados a las mujeres. En lugar del papel de la mujer como «cuidadora» del resto de la familia, se debería hacer más hincapié en su identidad más autónoma, en su rol de sujeto activo y positivo ${ }^{23,24}$.

En segundo lugar, una gran parte de las actividades propuestas en los programas funda- 
mentados en esta orientación más clásica iban destinadas a abrir el mundo de la mujer hacia un espacio "exterior» al hogar, espacio en el que las mujeres podían anudar nuevas relaciones sociales que las sacara de su aislamiento más tradicional. Sin embargo, la investigación del año 2000 pone de manifiesto la necesidad de programas y de actividades que faciliten a las mujeres unas mejores formas de gestión y organización del tiempo y que posibiliten, por otro lado, el desarrollo de la noción y de la práctica de los autocuidados. Un ejemplo cotidiano puede resultar significativo de esta evolución, mientras en 1993 se hablaba con tristeza del momento en el que los hijos dejaban el hogar, en relación con el síndrome del nido vacío ${ }^{25}$, en el 2000 se señalaba irónicamente la alegría que producía que los hijos se emancipasen y permitieran la liberación de los padres, unas nuevas posibilidades de uso de sus tiempos libres.

Tal como hemos tratado de representar en la figura 5, dicho cambio significa posicionar los programas de salud destinados a las mujeres en una situación más «personalizada», a caballo entre la noción de la «vida saludable», más holística, y la de los «hábitos saludables» más individualizados y normativizados. Posicionamiento más central que permite visualizar cómo estos programas deben fomentar los autocuidados en la doble dimensión de «cuidados físicos y psíquicos», frente a la idea más tradicional del «afuera» como espacio psíquicamente más saludable.

A modo de ejemplo, este nuevo posicionamiento más central de los programas de salud destinados a las mujeres podría traducirse en el desarrollo de los siguientes tipos de programas.

- En el espacio más próximo a una dimensión más física de la salud y desde posi- ciones próximas a la promoción de una cultura de hábitos saludables, parecería posible pensar en el desarrollo de programas específicos que puedan estar dirigidos a la prevención desde la alimentación-nutrición y desde el ejercicio físico/deporte. El desarrollo de posibles programas de salud laboral también podría inscribirse en este espacio más físico, en la medida en que se trataría de prevenir los posibles malestares o enfermedades ocasionados por el desempeño del trabajo, ya sea desde corrección de posiciones frente al ordenador, ya sean relacionadas con las condiciones de seguridad e higiene en el trabajo o con otras situaciones laborales.

— En un espacio más central, y en este sentido más próximo a la noción de salud que parece expresarse de forma dominante entre las mujeres madrileñas en el año 2000, la salud entendida como equilibrio personal e integral, los programas contra el estrés, como puedan ser las actividades de yoga o semejantes, parecen responder a la nueva realidad de la mujer. Programas en los que se combina de forma equilibrada el autocuidado del cuerpo y de la mente.

- Dentro del espacio próximo al cuidado de la dimensión psíquica de la salud, podrían promoverse también, tal vez más dirigidos a amas de casa, programas más relacionales más activos, programas que faciliten las relaciones sociales de las mujeres, como puedan ser circuitos «verdes», circuitos «culturales», junto al mantenimiento de los programas actuales que siguen teniendo sentido.

Conjunto de iniciativas de programas y posibles conjuntos de mujeres como sectores preferentes de los mismos que, por último, hemos tratado de recoger esquemáticamente en la figura 6 . 


\section{Figura 5}

Evolución tendencial del espacio central en el que se inscriben los programas de promoción de la salud Cimop 2001

\section{CUIDADOS FÍSICOS}

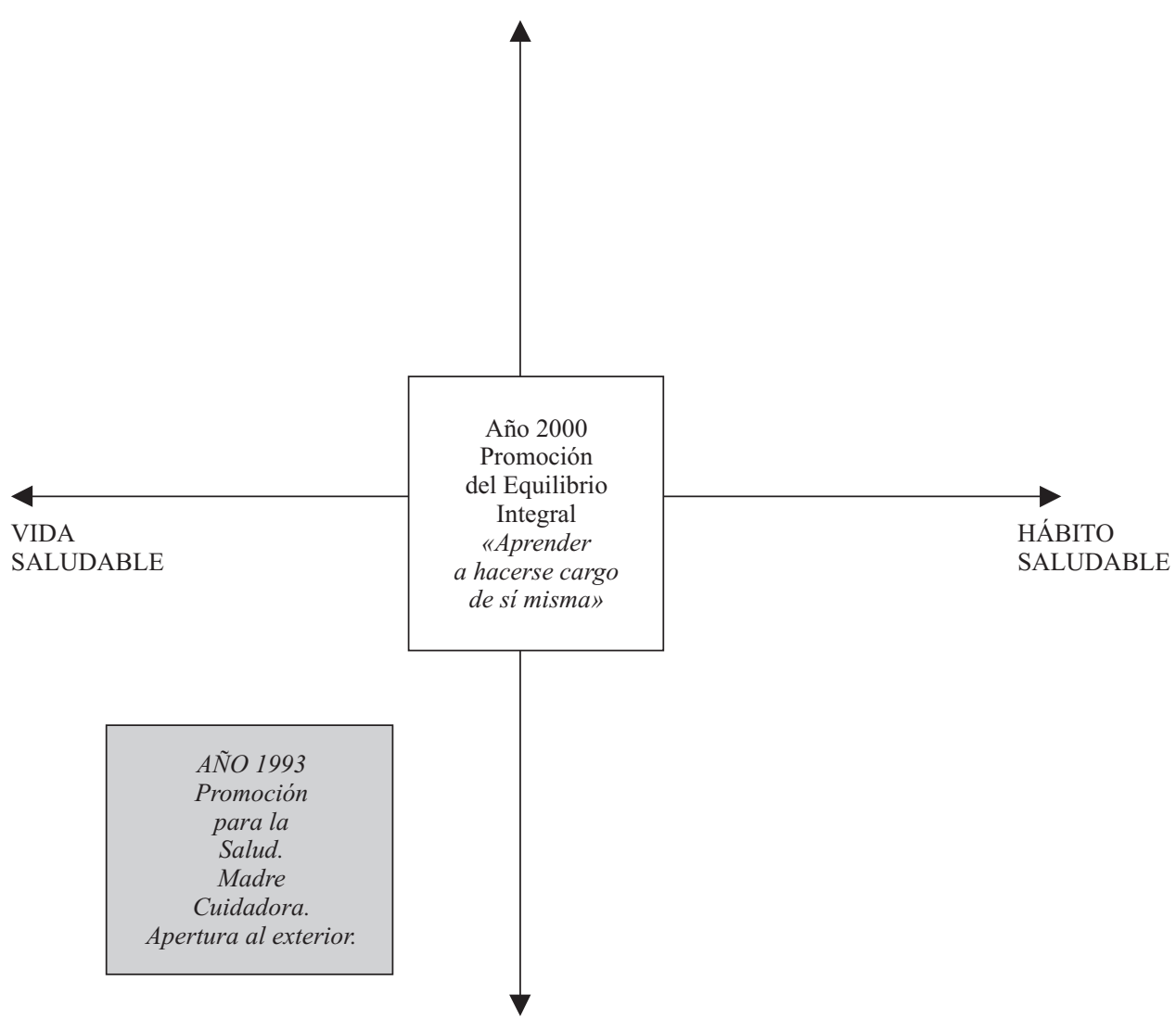

CUIDADOS PSÍQUICOS 
Figura 6

Cercanía de los colectivos de mujeres a los Programas de Promoción de la Salud

\section{CUIDADOS FÍSICOS}

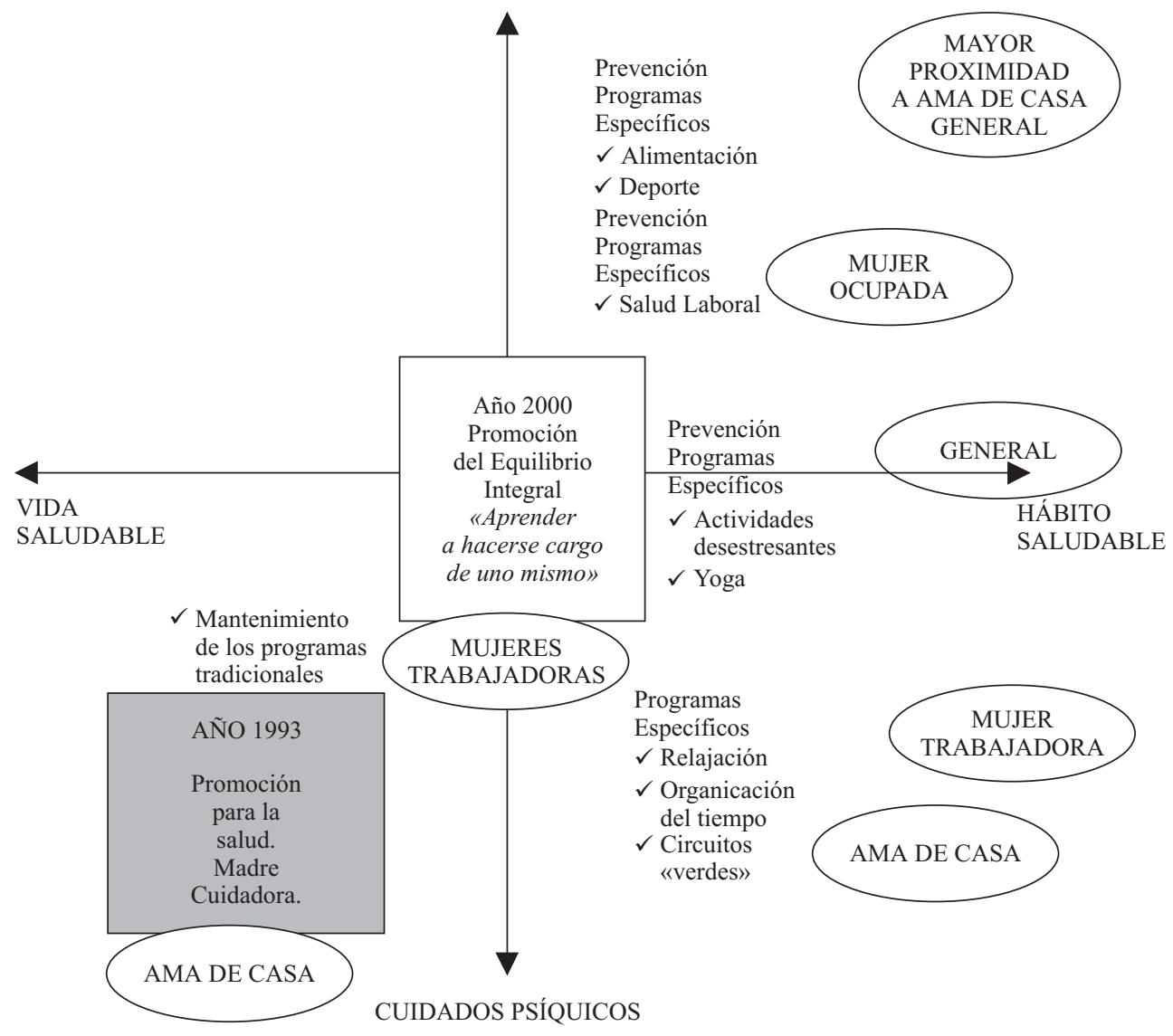

\section{BIBLIOGRAFÍA}

1. Gil Nebot M, Estrada Ballesteros C, Pires Alcalde ML y Aguirre Martín-Gil R. La investigación cualitativa y la promoción de la salud en la Comunidad de Madrid. Rev Esp Salud Publica 2002; 76: 451-459.

2. Ministerio de Sanidad y Consumo. Indicadores de Salud. Tercera evaluación en España del programa regional europeo Salud para todos. 1995 Madrid: Ministerio de Sanidad y Consumo; 1996.

3. Ander Egg. E. Técnicas de Investigación Social. Mejico: Ateneo; 1990.
4. Aracil Rodríguez E. y otros autores. Sistema Gráfico de Información Sanitaria en España. Barcelona: Grupo MSD; 1996.

5. Setien ML. Indicadores Sociales de Calidad de Vida. Un Sistema de medición aplicado al P Vasco. Madrid: CIS; 1993.

6. Las Ciudades saludables en Euskadi. Documentos Técnicos de Salud Pública. Vitoria-Gasteiz: Gobierno Vasco; 1990.

7. Doise W, Clemence A, Lorenzi-Cioldi F. Representations Sociales et Analyse des Données. Grenoble: P. Universitaires de Grenoble; 1992.

Rev Esp Salud Pública 2002, Vol. 76, N. ${ }^{\circ} 5$ 
8. Flick V. La Perception quotidienne de la santé et de la maladie. Theories Subjectives et Representations Sociales. Paris: L'Harmattan; 1992.

9. Conde F. Una propuesta de uso conjunto de las técnicas cuantitativas y cualitativas en la investigación social. El isomorfismo de las dimensiones topológicas de ambas técnicas. Revista Española de Investigaciones Sociológicas 1987; 39: 213-24.

10. Conde F. Un ensayo de articulación de las perspectivas cuantitativa y cualitativa en la investigación social. Revista Española de Investigaciones Sociológicas; 1991: 91-117.

11. Consejería de Sanidad y Servicios Sociales. Informe sobre la salud y la mujer en la Comunidad Autónoma de Madrid. Documentos Técnicos de salud Pública n. ${ }^{\circ}$ 32. Madrid: Consejería de Sanidad y Servicios Sociales; 1998.

12. Consejería de Sanidad y Servicios Sociales. Las Representaciones Sociales sobre la salud de los jóvenes madrileños. Documentos Técnicos de Salud Pública n. ${ }^{\circ}$ 45. Madrid: Consejería de Sanidad y Servicios Sociales; 1998.

13. Consejería de Sanidad y Servicios Sociales. Las Representaciones sociales sobre la salud de la población activa masculina de la Comunidad de Madrid.. Documentos Técnicos de Salud Pública n. ${ }^{\circ}$ 47. Madrid: Consejería de Sanidad y Servicios Sociales; 1998.

14. Consejería de Sanidad y Servicios Sociales. Las Representaciones Sociales sobre la salud de los niños de 6 a 12 años de la Comunidad de Madrid Documentos Técnicos de Salud Pública n. ${ }^{\circ} 48$. Madrid: Consejería de Sanidad y Servicios Sociales; 1998.

15. Consejería de Sanidad y Servicios Sociales. Las Representaciones Sociales sobre la Salud de los Mayores Madrileños. 1997. Documentos Técnicos de salud Pública n. ${ }^{\circ}$ 32. Madrid: Consejería de Sanidad y Servicios Sociales; 1998.
16. Martínez Ramos E. Aspectos Teóricos del Análisis Clusters y aplicación a la caracterización del electorado potencial de un partido. En: Introducción a las técnicas de análisis multivariable aplicadas a las ciencias sociales. Sánchez Carrión JJ editor. Madrid: CIS; 1984

17. Ortí A. La apertura y el enfoque cualitativo o estructural: la entrevista abierta semidirectiva y la discusión de grupo. En: El análisis de la realidad social. Métodos y técnicas de investigación. García Ferrando M, Ibáñez J y Alvira F editores. Madrid: Alianza Universidad; 1986.

18. Ibáñez J Cómo realizar una investigación mediante grupos de discusión» En: El análisis de la realidad social. Métodos y técnicas de investigación. García Ferrando M, Ibáñez J y Alvira F editores. Madrid: Alianza Universidad; 1986.

19. Cook T.D. y Reichardt Ch. Métodos Cualitativos y Cuantitativos en Investigación Evaluativa. Madrid: Morata; 1986.

20. Ricoeur P. Teoría de la Interpretación. Discurso y excedente de sentido. Madrid: Siglo XXI; 1995.

21. Thom. R. Parábolas y Catástrofes. Barcelona: Tusquets; 1985.

22. Benzecri JP. L'analyse des données. Paris: Dunod; 1982

23. Instituto de la Mujer. Vivir con salud, haciendo visibles las diferencias. Madrid: Instituto de la Mujer. Ministerio de Trabajo y Asuntos Sociales; 1997.

24. Conde F. El estrés en las mujeres trabajadoras: sus causas y sus consecuencias. Madrid: Instituto de la Mujer. Ministerio de Trabajo y Asuntos Sociales; 2000

25. Dio Bleichmar E. La Depresión en la Mujer. En: Cuerpo y Subjetividad Femenina. Salud y Género. González de Chávez MA editor. Madrid: Siglo XXI; 1993. 
